\title{
Semiological Features of Nonepileptic Paroxysmal Events in Infancy
}

\author{
Ha Rim Noh, MD, Young Hwan Kim, MD, Kye Hyang Lee, MD \\ Department of Pediatrics, Daegu Catholic University School of Medicine, Daegu, Korea
}

Received: August 30, 2019

Revised: November 5, 2019

Accepted: December 12, 2019

Corresponding author:

Kye Hyang Lee, MD

Department of Pediatrics, Daegu

Catholic University School of

Medicine, 33 Duryugongwon-ro

17-gil, Nam-gu, Daegu 42472,

\section{Korea}

Tel: +82-53-650-4242

Fax: +82-53-650-4243

E-mail: rosalia@cu.ac.kr
Purpose: Nonepileptic paroxysmal events (NPEs) are common in infancy and may be misinterpreted as epileptic seizures. With a knowledge of common NPEs, they can usually be diagnosed based on a detailed history and examination. So far, no studies have explored the semiological presentations of NPEs in infancy without video electroencephalographic (VEEG) recordings. We aimed to describe the phenomenology of NPEs in infancy to provide useful information to clinicians, enabling easier diagnoses without VEEG studies.

Methods: We reviewed the medical records of 63 patients aged from 1 to 12 months diagnosed with NPEs at Daegu Catholic University Medical Center from September 2006 to June 2017. We classified the phenomenological features into five types: abnormal body movement, eye changes, breathing abnormalities, behavioral symptoms, and autonomic symptoms.

Results: Of the 63 patients, 37 were male and 26 were female. The mean age at onset was 6 months, and the mean duration of symptoms was 22.5 days. Abnormal body movements were the most common feature (88.9\%), followed by eye changes (31.7\%), autonomic symptoms (11.1\%), breathing abnormalities (6.3\%), and behavioral abnormalities (3.2\%). The most common type of abnormal body movements was shivering-like movement (32.1\%). Initially, 30 patients (47.6\%) were diagnosed with unclassified NPEs, nine (14.3\%) with sleep myoclonus, six (9.5\%) with benign paroxysmal tonic upward gaze, and five (7.9\%) with benign myoclonus of infancy. During follow-up, two patients (3.2\%) were diagnosed with epilepsy.

Conclusion: Knowledge of the phenomenological characteristics of NPEs in infancy can be useful for making a correct diagnosis.

Keywords: Seizures; Dyskinesias; Infant

\section{Introduction}

Nonepileptic paroxysmal events (NPEs) are characterized by seizure-like behaviors without concomitant ictal electroencephalography (EEG), and common in infancy [1,2].

According to Visser et al. [3], the incidence of paroxysmal disorders in infancy is $8.9 \%$, of which $52 \%$ are physiological events.
NPEs may be misinterpreted as epileptic seizures, which is likely to cause alarm amongst parents and concern amongst professionals. The range of possible diagnoses for a paroxysmal disorder in infan$c y$ is extensive. A number of these conditions are benign and often physiological in many cases, such as neonatal tremor, benign neonatal sleep myoclonus, and shuddering attacks [2,4]. Accurate diagnosis is crucial for avoiding unnecessary evaluation and medica-

Copyright(C) 2020 Korean Child Neurology Society

This is an Open Access article distributed under the terms of the Creative Commons Attribution Non-Commercial License (http://creativecommons.org/licenses/by-nc/4.0/) which permits unrestricted non-commercial use, distribution, and reproduction in any medium, provided the original work is properly cited. 
tion, and alleviating parents' worries [2,5-7]. Diagnosis can usually be made based on detailed history and examination, with the knowledge of several types of NPEs with distinctive clinical presentations $[5,7]$. However, differentiation between true seizures and NPEs is difficult during infancy due to variations in semiology and incorrect history provided by parents or caregivers. Because of these difficulties, video electroencephalographic (VEEG) recording is required for a definitive diagnosis $[6,8]$. Although VEEG recording is the most accurate diagnostic method, it is difficult to perform in infants, and cannot easily be performed in all medical centers [5]. Due to widely available smartphones, mobile phone video can be used as an important diagnostic tool before proceeding to VEEG recording. Although there are several reports of NPEs with VEEG recordings in children, there are no reports of the various semiological presentations of NPEs in infancy without VEEG study.

We aimed to describe the phenomenology of NPEs in infants to provide clinicians with useful information for more accurate and easier diagnosis, without VEEG recording.

\section{Materials and Methods}

After Institutional Review Board approval (CR-17-086-L), we retrospectively reviewed the medical records of 63 children aged from 1 to 12 months, diagnosed with NPEs, or pseudoseizure, between September 2006 and June 2017 at Daegu Catholic University Medical Center. Written informed consent by the patients was waived due to a retrospective nature of our study. We analyzed sex, age, age at onset, duration of symptoms before seeking medical attention, frequency, form of main symptom, situation of occurrence, responsiveness to stimulation during the event, preceding event, gestational age, developmental history, family history, associated medical conditions, electrocardiogram (ECG), EEG, brain imaging, initial and final diagnosis. As for the frequency of events at the initial visit, we included even a single episode, because NPEs can happen as a single episode. Based on semiological features, the main symptoms were categorized into five types as follows: abnormal body movement, eye changes, breathing abnormalities, behavioral, and autonomic symptoms. Abnormal body movements included any type of facial or bodily movements. Eye changes included eye deviation, blinking, nystagmus, fixed pupils, and opening wide. Breathing abnormalities included apnea and irregular coarse respiration. Behavioral symptoms included staring and arrest of activity. Autonomic features included retching, drooling, facial color changes, and tachycardia. Although the phenomologic character was categorized by main symptoms, many patients had multiple simultaneous features. Therefore, we also analyzed all semiological descriptions. The situation of occurrence was categorized as follows: playing, sleeping, feeding, sleepy, awakening, specific position or crying. Responsiveness was defined as changes in the event upon stimulation. Additional work ups including blood tests, ECG, EEG, and brain imaging were performed when there was a clinical suspicion for seizures based on history. The initial diagnosis was made by the physician at the first encounter based on clinical information, and the final diagnosis was made at a later date, after a period of follow-up. Highly suspecting epileptic seizures were termed as epilepsy in the initial diagnosis lists.

\section{Definitions of conditions mimicking epileptic seizures}

- Apparent life-threatening events (ALTE) or brief resolved unexplained events: an episode that is frightening to the observer and characterized by some combination of apnea, color change, marked change in muscle tone, choking or gagging $[4,9]$.

- Benign paroxysmal vertigo (BPVC): migraine equivalent, consisting of episodes of vertigo lasting from a few seconds to minutes, that is often accompanied by postural imbalance and nystagmus [9].

- Startle response: also known as the startle reflex and the alarm reaction. The alarm reaction is a completely natural, involuntary reaction to a stimulus such as a flash of light, sudden threatening movement or loud noise $[4,7,9]$.

- Benign paroxysmal tonic upward gaze (PTU): protracted attacks (hours to days) of continuous or episodic upward gaze deviation, during which horizontal eye movements are preserved $[7,9,10]$.

- Sleep myoclonus: repetitive, usually bilateral rhythmic jerks involving the upper and lower limbs during nonrapid eye movement sleep, sometimes mimicking clonic seizures $[4,7,9,10]$.

- Benign myoclonus of infancy (BMI): myoclonic jerks of the extremities during wakefulness and sometimes also during sleep $[2,9]$.

- Shuddering attacks: rapid tremor of the head, shoulder, and trunk, lasting a few seconds, often associated with eating, and recurring many times a day [9].

- Benign paroxysmal torticollis of infancy (BPT): painless retrocollis, and later, torticollis, often triggered by changes in posture $[2,7,9,10]$.

- Unclassified NPEs: unclassified infantile behavior (age related behavior, pseudoseizure) [2].

\section{Data analyses}

This descriptive study used mean, maximum and minimum for the two continuous variables and frequency to describe discontin- 
uous variables.

\section{Results}

\section{Demographic and clinical characteristics}

Of the 63 patients, 37 (58.7\%) were male and 26 (41.3\%) were female, mean age at onset was $5.6 \pm 2.9$ months (range, 1 to 11 ), and mean duration of symptoms was $22.5 \pm 28.3$ days (range, 1 to $90)$. Each one (1.6\%) had one of the following preceding events before symptom onset: vaccination, febrile seizure, and bronchiolitis. Family history of febrile seizure, epilepsy, and migraine was each present in one patient (1.6\%). Ten patients (16\%) had associated medical conditions, including developmental delay, constipation, bilateral sensorineural hearing losses with hypotonia, the chromosomal abnormality 47, XY,+der(22)t(11;22)(q23.3; q11.2), laryngomalacia, febrile seizure, hemophilia, or periventricular leukomalacia.

In 27 infants (42.9\%), events occurred daily. Twenty-three (37\%) showed responsiveness to external stimuli. Events most commonly occurred when playing (58.7\%). Twenty-four patients (38.1\%) underwent EEG and four (6.3\%) showed abnormal results. Neuroimaging was performed in five patients (7.9\%), and none had abnormal findings (Table 1).

\section{Phenomenology of nonepileptic paroxysmal events}

According to main symptom categories, abnormal body move-

Table 1. Clinical characteristics of 63 patients

\begin{tabular}{lc}
\hline Characteristic & Value \\
\hline Frequency of events & $13(20.6)$ \\
One or two & $27(42.9)$ \\
Daily & $22(34.9)$ \\
Occasionally & \\
Situation of events & $43(68.2)$ \\
Playing & $11(17.4)$ \\
Sleeping & $7(11.1)$ \\
Feeding & $6(9.5)$ \\
When feeling sleepy & $1(1.6)$ \\
Awakening & $3(4.8)$ \\
Specific position/crying & $24(38.1)$ \\
Electroencephalography & $4(16.6)$ \\
Abnormal & 1 \\
$\quad$ Epileptic discharge & 3 \\
Slow wave & $5(7.9)$ \\
Brain imaging & $2(3.2)$ \\
Electrocardiogram &
\end{tabular}

Values are presented as number (\%). ment was the most common and present in 50 patients (79.4\%). Patient numbers in the other categories were as follows: seven had eye changes (11.1\%), four had breathing abnormalities (6.4\%), one had behavioral symptoms (1.6\%), and one had autonomic symptoms (1.6\%). Among 63 patients, 15 (23.8\%) had a mixture of symptoms from different categories. Overall, 56 patients (88.9\%) had abnormal body movements, 20 patients (31.7\%) had eye changes, and four patients $(6.3 \%)$ had breathing abnormalities. Seven patients (11.1\%) had autonomic symptoms, with facial color change being the most common. Staring was the only behavioral symptom observed, occurring in two patients (3.2\%). Two patients (3.2\%) had vocalization symptoms such as making 'mmmm' sounds and shouting, which were accompanied by other symptoms.

For the phenomena of abnormal body movements, shivering-like movement was the most common (18/56 patients, $32.1 \%)$. For ocular symptoms, eyeball deviation was the most common (10/20 patients, $50.0 \%)$, and six patients ( $6 / 20$ patients, $30.0 \%$ ) had isolated eye changes (Tables 2 and 3 ).

\section{Initial and final diagnoses of NPEs}

For the initial diagnoses, 30 patients (47.6\%) were diagnosed with unclassified NPEs, nine (14.3\%) with sleep myoclonus, six (9.5\%) with PTU, five (7.9\%) with BMI, three (4.8\%) with ALTE, two (3.2\%) with shuddering attack, two (3.2\%) with BPVC, one

Table 2. Clinical phenomenology of abnormal body movements

\begin{tabular}{lc}
\hline Variable & Value \\
\hline Abnormal body movements & $56 / 63(88.9)$ \\
Shivering-like movement & $18 / 56(32.1)$ \\
Stiffening & $10 / 56(17.9)$ \\
Head shaking side to side & $6 / 56(10.7)$ \\
Myoclonic, startle & $6 / 56(10.7)$ \\
Clonic & $3 / 56(5.4)$ \\
Opisthotonic posturing & $2 / 56(3.6)$ \\
Shrinking & $2 / 56(3.6)$ \\
Head nodding forward & $2 / 56(3.6)$ \\
Irregular movement of the extremities & $2 / 56(3.6)$ \\
Chin movement & $2 / 56(3.6)$ \\
Atonic, limping & $2 / 56(3.6)$ \\
Ataxic & $2 / 56(3.6)$ \\
Torticollis & $2 / 56(3.6)$ \\
Shoulder shrugging & $2 / 56(3.6)$ \\
Facial grimacing & $2 / 56(3.6)$ \\
Tortipelvis & $1 / 56(1.8)$ \\
Akinetic, frozen, still & $1 / 56(1.8)$
\end{tabular}

Values are presented as number (\%). 
Table 3. Clinical phenomenology of other categories

\begin{tabular}{lc}
\hline Variable & Value \\
\hline Eye changes & $20 / 63(31.7)$ \\
Deviation & $10 / 20(50.0)$ \\
Opening wide & $4 / 20(20.0)$ \\
Blinking & $2 / 20(10.0)$ \\
Fixed & $2 / 20(10.0)$ \\
Nystagmus & $1 / 20(5.0)$ \\
Mixed & $1 / 20(5.0)$ \\
Autonomic nervous system & $7 / 63(11.1)$ \\
Facial color change (flushing, pallor) & $4 / 7(57.1)$ \\
Digestive symptoms (retching, drooling) & $2 / 7(28.6)$ \\
Cardiovascular (tachycardia) & $1 / 7(14.3)$ \\
Breathing abnormalities & $4 / 63(6.3)$ \\
Apnea & $3 / 4(75.0)$ \\
Irregular and coarse breathing & $1 / 4(25.0)$ \\
Vocalization & $2 / 63(3.2)$ \\
Behavioral staring & $2 / 63(3.2)$
\end{tabular}

Table 4. Initial and final diagnosis of 63 patients with nonepileptic paroxysmal events

\begin{tabular}{lcc}
\hline Variable & Initial & Final \\
\hline Unclassified NPEs & $30(47.6)$ & $35(55.6)$ \\
Sleep myoclonus & $9(14.3)$ & $9(14.3)$ \\
PTU & $6(9.5)$ & $5(7.9)$ \\
BMI & $5(7.9)$ & $6(9.5)$ \\
ALTE & $3(4.8)$ & $2(3.2)$ \\
Shuddering attack & $2(3.2)$ & $2(3.2)$ \\
BPVC & $2(3.2)$ & $1(1.6)$ \\
BPT & $1(1.6)$ & $1(1.6)$ \\
Startle response & $1(1.6)$ & $0(0.0)$ \\
Epilepsy & $4(6.3)$ & $2(3.2)$
\end{tabular}

Values are presented as number (\%).

NPE, nonepileptic paroxysmal event; PTU, benign paroxysmal tonic upward gaze; BMI, benign myoclonus of infancy; $A L T E$, apparent life-threatening events; BPVC, benign paroxysmal vertigo; $B P T$, benign paroxysmal torticollis of infancy.

Values are presented as number (\%).

Table 5. Characteristics of two patients finally diagnosed with epilepsy

\begin{tabular}{lll}
\hline Characteristic & \multicolumn{1}{c}{ Patient 1 } & Patient 2 \\
\hline Age of onset & 6 months & 5 months \\
Phenomenology & Apneic episode with limping, blank stare followed by pallor Sudden upward gaze \\
with dilated pupils and body shaking movements & \\
Frequency & Once & Daily \\
Duration & 1 day & 60 days \\
During sleep & Yes & No \\
Responsiveness & Unknown & Unknown \\
Gestational age & Full term & Full term \\
Developmental delay & No & No \\
Past medical history & Febrile seizure & No \\
EEG & Yes, IS in the left temporal & Not done \\
Brain MRI & Yes & Not done \\
Initial diagnosis & ALTE & PTU \\
Course & Three focal seizures with impaired awareness developed at & After 1 month, head nodding with flexor spasm developed \\
Final diagnosis & 3 and 10 months after initial events & \\
\hline
\end{tabular}

EEG, electroencephalography; IS, intermittent slow; MRI, magnetic resonance imaging; ALTE, apparent life-threatening events; PTU, paroxysmal tonic upward gaze.

(1.6\%) with BPT, one (1.6\%) with startle response, and four (6.3\%) with epilepsy. After the study and follow-up observations, the initial diagnosis was changed in some patients (Table 4). During follow-up, two patients (3.2\%) were diagnosed with epilepsy. One patient was initially diagnosed with PTU, then finally with West syndrome. We summarized the characteristics of the two cases finally diagnosed as epilepsy (Table 5).

\section{Discussion}

The frequency of NPEs in children admitted to epilepsy centers for monitoring ranges between 3.5\% and 43\% [1]. As VEEG studies require frequent occurrences of an event and are also very demanding, especially for infants, every effort should be made to reach a diagnosis based on clinical symptoms [6].

Our study provides a detailed description of the phenomenolo- 
gy of NPEs in infants, and useful clinical tips for their differential diagnosis based on clinical information without VEEG study. Because phenomenological features of NPEs in infancy are varied and complex, they are usually classified arbitrarily according to main clinical features even with VEEG study data $[5,10]$.

Therefore, we categorized NPEs in infancy into five types; movement, eye, breathing, behavioral, and autonomic. For comparison with previous studies and discussion purposes, we classified breathing as an autonomic symptom, and eye changes and vocalization as motor symptoms.

\section{Clinical phenomena}

\section{1) Motor symptoms}

Motor phenomena, with paroxysmal features that interrupt normal motor background activity, are frequently observed in infants [7]. Chen et al. [5] found motor symptoms were the most common manifestation of NPEs in children younger than 2 years of age (68.4\%). Kim et al. [11] reported that tonic posturing was the most common NPE in infancy, followed by staring. Park et al. [8] also analyzed NPEs in children younger than 6 years of age and found that normal infant behavior (29.2\%) was most commonly observed. Moreover, normal infant behaviors, such as repetitive grimacing, mouth twisting, crossing legs with cold sweats, and tonic-clonic arm movements, were prominent in this age group. In the present study, we found abnormal body movement was the most common and present in $89 \%$ of patients, which is similar to previous studies. Among them, shivering-like movement was the most common. In clinical practice, there are clues to discriminate epileptic from nonepileptic motor events. Motor symptoms in epileptic seizures are predominantly deviations of the eyes and head, dystonic and tonic posturing of the limbs, clonic, tonic, or myoclonic jerks, and epileptic spasms that account for around $25 \%$ of the epileptic symptoms in this age group. Focal motor features, such as version, are not reported in NPEs. In contrast, the motor features in NPEs are inconsistent and include bizarre, irregular, jerking or thrashing movements of the extremities, generalized jerking/flashing, focal motor activity, complex motor activity, generalized tremor, head-shaking, generalized limpness, eye fluttering/visual blurring, and oromotor activity [5].

Myoclonus is another clinical motor feature that occurs in infants and toddlers with NPEs [5]. According to Fogarasi et al. [12], the presence of nocturnal myoclonus, only during sleep, should encourage the physician toward a diagnosis of NPEs, because children with epilepsy $\leq 2.6$ years of age do not have nocturnal symptom predominance, in the absence of a significant medical history.

Eye changes were a major category in our study and $32.1 \%$ had ocular symptoms. Among them, eye deviation was the most com- mon. However, there are no published studies describing this phenomenon in detail. Chen et al. [5] described eye changes as motor symptoms and reported three patients $(7.9 \%)$ with ocular changes such as eye closure, rolling, and blinking.

There were two patients (3.2\%) with vocalization in our study. According to Chen et al. [5], oral/verbal features are present in $36.8 \%$ of patients, crying (57.1\%) being the most common followed by vocalization and sighing. These features have been overlooked in young infants. Although crying during seizures is a wellknown feature of infantile spasms, Shuper and Mimouni [6] reported crying in one infant with NPEs, not epilepsy.

\section{2) Behavioral, staring events}

Behavioral features have been previously described in $10.4 \%$ to $25.5 \%$ of children with NPEs, but mainly in the school-aged group and teenagers. Nonepileptic staring spells and arrest of activity are frequent symptoms during NPEs in infants and toddlers as well [5].

Kim et al. [11] reported that staring was present in $5.7 \%$ of infants with NPEs. Staring was present in only two patients (3\%) in our study. One patient with autonomic features was diagnosed with epilepsy 10 months later.

Arrest of activity is also a prominent feature of hypomotor seizures in infants and toddlers. Characteristics of hypomotor seizures in this age group are as follows: (1) progression to other focal motor features, generalized seizures, or infantile spasms, and (2) subtle concomitant features, such as chewing or lip smacking, unforced eye movements, head turning, subtle irregular eye blinking, and stereotypical limb movements.

When compared to epileptic seizures, duration of nonepileptic staring was usually brief ( $<30$ seconds), and children were mostly responsive without crying.

\section{3) Autonomic symptoms}

Fogarasi et al. [13] showed that autonomic symptoms are common in childhood focal epilepsies, also appearing in infants and young children. Apnea/bradypnea occurs more frequently in younger children. Autonomic symptoms in infancy are probably overlooked by the observer, rather than absent, given the predominance of motor features [5].

Chen et al. [5] reported that autonomic features were present in $21.1 \%$ of patients with NPEs. In most cases, autonomic features accompanied other clinical features, in particular motor and oral/ verbal features. Seven patients (11.1\%) had autonomic symptoms in our study. All cases included other features similar to those previously reported [5]. We classified breathing abnormalities as an isolated category, although Chen et al. [5] categorized these as au- 
tonomic features. Eleven patients (17.4\%) had autonomic symptoms, if breathing abnormalities were to be considered autonomic symptoms.

\section{Diagnosis}

Visser et al. [3] subgrouped paroxysmal events into the broad categories of physiological events, seizures, loss of consciousness, apneic spells, parasomnias, other, and unknown. A large proportion of the disorders (51\%) were classified as 'physiological events,' which they defined as 'those that are consistent with the normal pattern of behavior of children that age.' Chen et al. [5] reported that nonepileptic staring spells and sleep myoclonus were the two most common NPEs in infant and toddlers. Our study revealed that unclassified NPEs was the most common final diagnosis followed by myoclonus, such as benign sleep myoclonus, or BMI. Four infants (6.3\%) were initially diagnosed with epilepsy based on clinical information. However, two patients (3.2\%) were finally diagnosed with epilepsy, which had a lower incidence than initial impressions. Considering the number of VEEG carried out for NPEs, these findings suggest that detailed clinical information is adequate for making a diagnosis without exacting study [1].

There were several limitations in making a final diagnosis. Because we could only assume some of the conditions mimicking epileptic seizure based on clinical history reported by parents, it was difficult to make a correct diagnosis even after obtaining EEG data. In addition, as we did not follow patients after the initial encounter and presumptive diagnosis, we could not be sure how long NPEs had actually lasted, or whether they had progressed to epilepsy or other neurological problems. Shuper and Mimouni [6] described the clinical phenomenology and resolution time in 13 NPEs patients, reporting that NPEs were present for a period of 2 weeks to 7 months. Based on these data, several weeks of clinical follow-up are recommended, because a significant number of spells will resolve spontaneously. If there is no improvement, further studies are needed, including video EEG recording. Therefore, we strongly recommend some period of observation in order to make a correct final diagnosis.

With advancements in technology such as YouTube and smartphones, it has become easier to observe paroxysmal events of concern, and instruct or educate parents. Moreover, mutations in several genes have recently been associated with some NPEs, such as calcium voltage-gated channel subunit alpha1 A (CACNA1A) in BPT and PTU, glycine receptor subunit alpha-1 (GLRA1) in hyperekplexia, and sodium voltage-gated channel alpha subunit 9 (SCN9A) in paroxysmal extreme pain disorde $[2,14,15]$. With genetic testing, it is possible to make more accurate diagnoses in clinical practice.
Therefore, we propose that knowledge of NPEs and the differentials between epileptic and nonepileptic events in infancy is useful for guiding clinicians in making the next decision, while avoiding exacting tests, in an outpatient setting.

\section{Conflicts of interest}

No potential conflict of interest relevant to this article was reported.

\section{ORCID}

Ha Rim Noh, https://orcid.org/0000-0002-0876-3024

Kye Hyang Lee, https://orcid.org/0000-0001-6646-8982

\section{Author contributions}

Conceptualization: KHL. Data curation: HRN and YHK. Formal analysis: HRN, YHK, and KHL. Methodology: HRN, YHK, and KHL. Project administration: KHL. Visualization: HRN, YHK, and KHL. Writing-original draft: HRN. Writing-review \& editing: KHL.

\section{Acknowledgements}

This study was presented as an oral presentation at the 67th Annual Autumn Meeting of the Korean Pediatric Society, 2017.

\section{References}

1. Kutluay E, Selwa L, Minecan D, Edwards J, Beydoun A. Nonepileptic paroxysmal events in a pediatric population. Epilepsy Behav 2010;17:272-5.

2. Besag FM, Hughes EF. Paroxysmal disorders in infancy: a diagnostic challenge. Dev Med Child Neurol 2010;52:980-1.

3. Visser AM, Jaddoe VW, Arends LR, Tiemeier H, Hofman A, Moll HA, et al. Paroxysmal disorders in infancy and their risk factors in a population-based cohort: the Generation R Study. Dev Med Child Neurol 2010;52:1014-20.

4. Cross JH. Differential diagnosis of epileptic seizures in infancy including the neonatal period. Semin Fetal Neonatal Med 2013;18:192-5.

5. Chen L, Knight EM, Tuxhorn I, Shahid A, Luders HO. Paroxysmal non-epileptic events in infants and toddlers: a phenomenologic analysis. Psychiatry Clin Neurosci 2015;69:351-9.

6. Shuper A, Mimouni M. Problems of differentiation between epilepsy and non-epileptic paroxysmal events in the first year of 
life. Arch Dis Child 1995;73:342-4.

7. Orivoli S, Facini C, Pisani F. Paroxysmal nonepileptic motor phenomena in newborn. Brain Dev 2015;37:833-9.

8. Park EG, Lee J, Lee BL, Lee M, Lee J. Paroxysmal nonepileptic events in pediatric patients. Epilepsy Behav 2015;48:83-7.

9. Fernandez-Alvarez E. Transient benign paroxysmal movement disorders in infancy. Eur J Paediatr Neurol 2018;22:230-7.

10. Mikati MA, Obeid MM. Conditions that mimic seizures. In: Kliegman RM, Stanton BF, St Geme JW, Schor NF, editors. Nelson textbook of pediatrics. 20th ed. Philadelphia: Saunders Elsevier; 2016. p. 2857-63.

11. Kim SH, Kim H, Lim BC, Chae JH, Kim KJ, Hwang YS, et al. Paroxysmal nonepileptic events in pediatric patients confirmed by long-term video-EEG monitoring: single tertiary center re- view of 143 patients. Epilepsy Behav 2012;24:336-40.

12. Fogarasi A, Janszky J, Faveret E, Pieper T, Tuxhorn I. A detailed analysis of frontal lobe seizure semiology in children younger than 7 years. Epilepsia 2001;42:80-5.

13. Fogarasi A, Janszky J, Tuxhorn I. Autonomic symptoms during childhood partial epileptic seizures. Epilepsia 2006;47:584-8.

14. Rosman NP, Douglass LM, Sharif UM, Paolini J. The neurology of benign paroxysmal torticollis of infancy: report of 10 new cases and review of the literature. J Child Neurol 2009;24:15560.

15. Kartal A. Paroxysmal tonic upgaze in children: three case reports and a review of the literature. Pediatr Emerg Care 2019; 35:e67-9. 